\title{
Parametrização e usabilidade do Sistema de Processo de Enfermagem
}

\author{
Parameterization and usability of the Nursing Process System \\ Parametrización y usabilidad del Sistema de Procesos de Enfermería
}

Recebido: 24/01/2022 | Revisado: 01/02/2022 | Aceito: 05/02/2022 | Publicado: 09/02/2022

Luciana Simões de Melo

ORCID: https://orcid.org/0000-0003-3017-6755 Universidade Federal de Sergipe, Brasil

E-mail:1smelo10@hotmail.com

Franciele Menezes Santana

ORCID: https://orcid.org/0000-0002-7455-3447 Universidade Federal de Sergipe, Brasil E-mail: francielemsantana@gmail.com

Jessika Valeska Martins Ramos

ORCID: https://orcid.org/0000-0001-7449-3694

Hospital Unimed Sergipe, Brasi

E-mail: jessikaramosenfa@hotmail.com

Clara Santana Sousa

ORCID: https://orcid.org/0000-0001-9326-1768

Empresa Brasileira de Serviços Hospitalares, Brasil E-mail: clarasantanasousa@gmail.com

Flávia Janólio Costacurta Pinto da Silva

ORCID: https://orcid.org/0000-0002-9866-5654 Universidade Federal de Sergipe, Brasil E-mail: fjanolio@gmail.com

Adicinéia Aparecida de Oliveira

ORCID: https://orcid.org/0000-0002-1551-1992 Universidade Federal de Sergipe, Brasil E-mail: adicineia@dcomp.ufs.br

Plínio Lima Barreto

ORCID: https://orcid.org/0000-0002-7728-0878 Universidade Federal de Sergipe, Brasil E-mail: plinio.ufs@gmail.com

Joseilze Santos de Andrade

ORCID: https://orcid.org/0000-0003-0488-2840 Universidade Federal de Sergipe, Brasil E-mail: joseilzesa@gmail.com

\begin{abstract}
Resumo
O presente estudo objetiva descrever a parametrização e usabilidade do Sistema de Processo de Enfermagem, um aplicativo web formulado para o registro das etapas do Processo de Enfermagem, por meio das linguagens padronizadas da enfermagem da NANDA-I, NIC e NOC. O processo de enfermagem orienta a documentação da prática profissional da enfermagem e se organiza em cinco etapas interdependentes e recorrentes. Realizou-se um estudo metodológicodescritivo, com a participação de 10 enfermeiros e 10 acadêmicos do último ano do curso de enfermagem atuantes em um hospital universitário, os quais avaliaram a funcionabilidade e usabilidade do SISPE. Após a inserção no sistema dos campos para a coleta de dados, dos diagnósticos, intervenções e resultados de enfermagem, foi possível verificar o registro do processo de enfermagem pelo SISPE e a vinculação entre suas etapas. A usabilidade do sistema obteve o escore médio da escala SUS de 77, 2, sendo avaliado como excelente. Conclusões: a parametrização do SISPE permitiu o registro das etapas do processo de enfermagem e a usabilidade do sistema foi avaliada como excelente.
\end{abstract}

Palavras-chave: Enfermagem; Processo de enfermagem; Software.

\begin{abstract}
The present study aims to describe the parameterization and usability of the Nursing Process System, a web application formulated to record the steps of the Nursing Process, through the standardized nursing languages of NANDA-I, NIC and NOC. The nursing process guides the documentation of professional nursing practice and is organized into five interdependent and recurring stages. A methodological-descriptive study was carried out, with the participation of 10 nurses and 10 students in the last year of the nursing course working at a university hospital, who evaluated the functionality and usability of SISPE. After inserting the fields for data collection, diagnoses, interventions and nursing results into the system, it was possible to verify the registration of the nursing process by SISPE and the link between its stages. The usability of the system obtained an average score on the SUS scale of 77.2, being evaluated as excellent.
\end{abstract}


Conclusions: the parameterization of SISPE allowed the recording of the steps of the nursing process and the usability of the system was evaluated as excellent.

Keywords: Nursing; Nursing process; Software.

\section{Resumen}

El presente estudio tiene como objetivo describir la parametrización y usabilidad del Sistema de Procesos de Enfermería, una aplicación web formulada para registrar los pasos del Proceso de Enfermería, a través de los lenguajes de enfermería estandarizados de NANDA-I, NIC y NOC. El proceso de enfermería orienta la documentación de la práctica profesional de enfermería y está organizado en cinco etapas interdependientes y recurrentes. Se realizó un estudio metodológicodescriptivo, con la participación de 10 enfermeras y 10 estudiantes del último año de la carrera de enfermería que laboran en un hospital universitario, quienes evaluaron la funcionalidad y usabilidad del SISPE. Después de insertar los campos de recolección de datos, diagnósticos, intervenciones y resultados de enfermería en el sistema, fue posible verificar el registro del proceso de enfermería por el SISPE y el vínculo entre sus etapas. La usabilidad del sistema obtuvo un puntaje promedio en la escala SUS de 77,2, siendo evaluado como excelente. Conclusiones: la parametrización del SISPE permitió el registro de los pasos del proceso de enfermería y la usabilidad del sistema fue evaluada como excelente.

Palabras clave: Enfermería; Proceso de enfermería; Software.

\section{Introdução}

O Sistema de Processo de Enfermagem (SISPE) é um sistema de informação para registro do Processo de Enfermagem (PE) formulado para utilização com base nos Sistemas de Classificação em Enfermagem (Souza, 2016).

O PE está respaldado na resolução do Conselho Federal de Enfermagem n 358 de 2009 e deve estar pautado por um processo técnico, organizado, deliberado e sistemático, a ser desenvolvido em todos os ambientes onde ocorra o cuidado profissional de enfermagem (COFEN, 2019).

Entende-se o processo de enfermagem como um guia sistemático que orienta a documentação da prática profissional e norteia o raciocínio diagnóstico e terapêutico do enfermeiro. Diagnósticos, intervenções e resultados de enfermagem são elementos da prática profissional que compõem o PE e podem ser documentados através de linguagem padronizada, de acordo com os Sistemas de Classificação em Enfermagem (Barros et al., 2020).

Dentre os vários Sistemas de Classificação em Enfermagem citam-se a Classificação dos Diagnósticos de Enfermagem da NANDA International, Inc. (NANDA-I), A Classificação dos Resultados de Enfermagem (NOC) e a Classificação das Intervenções de Enfermagem (NIC) (Herdman \& Kamitsuru, 2018; Moorhead et al., 2016; Bulecheck, 2016).

No contexto do cuidado em saúde, novas tecnologias são incorporadas ao processo de trabalho, neste caso, o uso dos Sistemas de Informação em Saúde visa contribuir para a implantação do PE informatizado a fim de facilitar sua aplicação, minimizar erros e apoiar a tomada de decisão do enfermeiro (Guimarães et al, 2020).

A usabilidade desses sistemas envolve a participação do usuário e a capacidade de executar tarefas por meio deles. A utilização da informática na Enfermagem auxilia na integração de pessoas, informações, procedimentos e recursos de computação, permitindo maior qualidade da assistência e consequentemente maior satisfação e segurança para o cliente (Rosa, 2019).

Sistemas informatizados permitem ao profissional o registro de dados e a comunicação interdisciplinar mais rápida e eficaz, portanto constituem importante ferramenta para o aprimoramento do cuidado prestado ao paciente (Oliveira et al., 2021).

Para medir a usabilidade do SISPE optou-se por utilizar a escala de usabilidade de sistemas (SUS, do inglês System Usability Scale), um instrumento amplamente utilizado por ser simples e rápido em sua aplicação. Os testes de usabilidades avaliam a qualidade da interação entre usuários e sistemas, possibilitando identificar problemas de usabilidade, medir o impacto destes e apontar suas causas, de modo que venham a ser corrigidos (Augusto et al.2021). 
Já a parametrização do SISPE ocorreu pela inserção de linguagens padronizadas da enfermagem, de modo que viesse a ocorrer o registro da anamnese, diagnósticos, planejamento, intervenções e resultados de enfermagem, ou seja, tornando possível o registro das cinco etapas do PE no sistema.

Esse estudo pretendeu contribuir com a inserção das tecnologias de informação na prática profissional e na formação de enfermeiros, possibilitando enfatizar o processo de enfermagem por meio de uma ferramenta interativa e intuitiva. Dessa forma, os objetivos desta pesquisa foram descrever a parametrização do SISPE e avaliar sua usabilidade.

\section{Delineamento do Estudo}

Foi realizado um estudo metodológico-descritivo. A escolha pelo esse método foi fundamentada em Polit e Beck (2019), os quais afirmam que este tipo de estudo tem a intenção de verificar o desenvolvimento, validar e avaliar ferramentas ou estratégias metodológicas.

O teste de funcionabilidade do SISPE ocorreu em um hospital universitário da região nordeste do Brasil e dele participaram 10 enfermeiros da unidade de terapia intensiva do referido hospital e 10 estudantes do último período da graduação do curso de enfermagem.

\subsection{Etapas da pesquisa}

\subsubsection{Parametrização do Sistema de Processo de Enfermagem}

Realizada com base na teoria das necessidades humanas básicas e nas taxonomias NANDA-I, NIC e NOC. Foram inseridos campos para registro da coleta de dados, organizados segundo a Teoria das Necessidades Humanas Básicas (NHB) de Wanda Horta de Aguiar. Depois foram cadastrados diagnósticos, intervenções, atividades e resultados de enfermagem.

\subsubsection{Avaliação da Usabilidade do Sistema}

Após utilizar o SISPE os participantes do estudo avaliaram sua usabilidade através da aplicação da escala SUS.

\section{Resultados e Discussão}

\subsection{Parametrização do Sistema de Processo de Enfermagem}

Nesta etapa do estudo foram inseridos no sistema os elementos que compõem a anamnese de enfermagem e os diagnósticos, intervenções, atividades e resultados de enfermagem, utilizando as linguagens padronizadas da enfermagem Nanda-I, NIC e NOC.

Para que ocorressem os relacionamentos entre as fases do PE, pela conexão entre as linguagens padronizadas da enfermagem, a inserção dos componentes da parametrização iniciou a partir dos resultados de enfermagem. Depois inseriu-se as intervenções e atividades de enfermagem, diagnósticos de enfermagem e coleta de dados, conforme descrito na Figura 1. 
Figura 1 - Etapas da parametrização do SISPE.

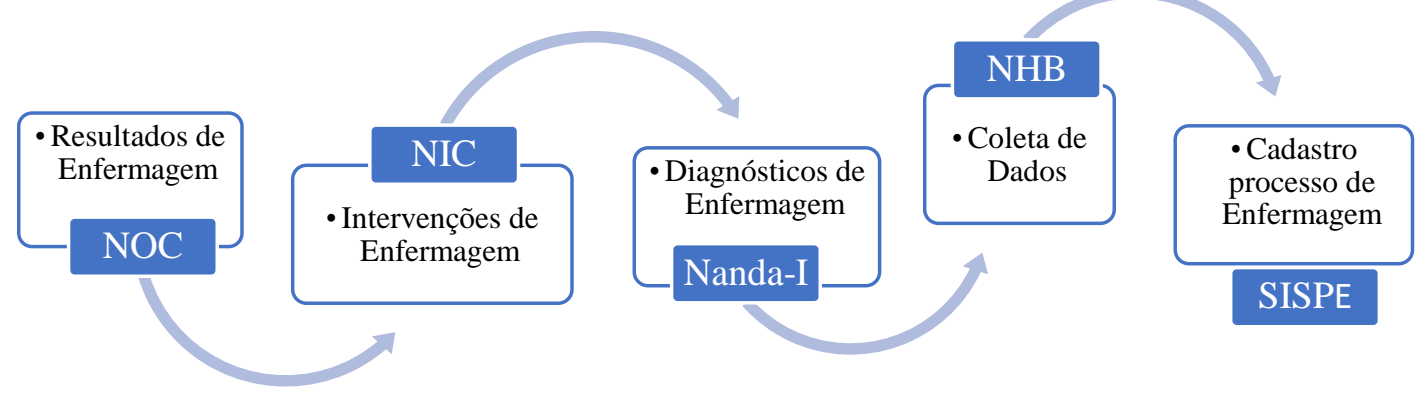

Fonte: Autores (2021).

Nesta etapa do estudo utilizou-se um roteiro instrucional composto por diagnósticos, intervenções, atividades e resultados de enfermagem, elaborado com as taxonomias complementares da Nanda-I, NIC e NOC, com base em diagnósticos de enfermagem frequentes na terapia intensiva.

Vinte e nove resultados de enfermagem e sessenta e sete indicadores relacionados foram cadastrados. Para cada resultado o usuário do sistema irá selecionar os indicadores da NOC mais condizentes com o perfil do paciente que está sendo avaliado. A Figura 2 demonstra a lista dos resultados de enfermagem cadastrados no SISPE.

Figura 2 - Lista dos Resultados Esperados/Obtidos cadastrados no SISPE.

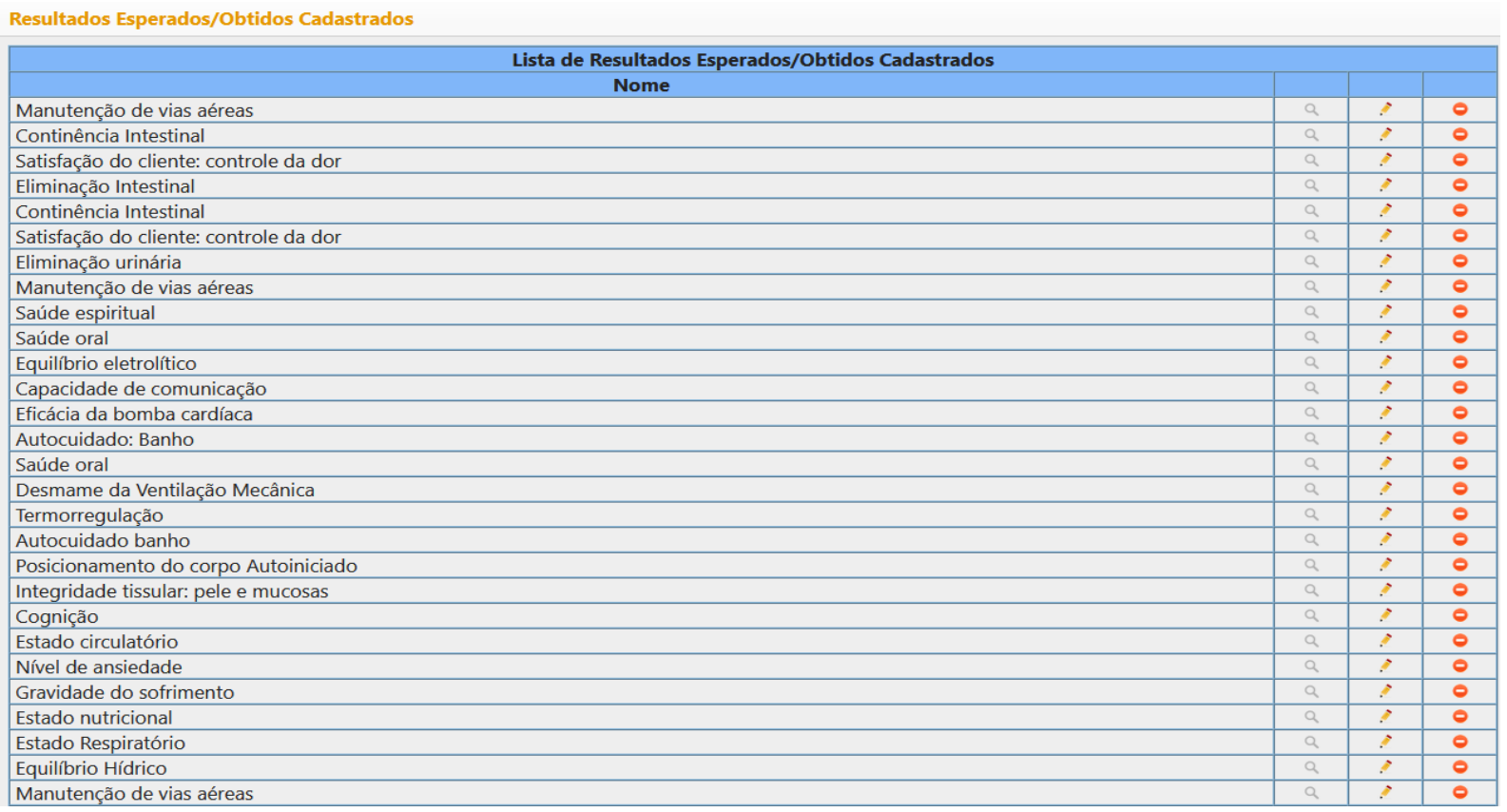

Fonte: SISPE (2021).

Após a inserção dos resultados foram lançadas no SISPE cento e oitenta e nove atividades e vinte e nove intervenções de enfermagem. Ao cadastro de cada atividade inseriu-se os resultados de enfermagem relacionados, de modo que ao término dessa etapa o sistema pôde estabelecer a conexão entre resultados e intervenções/atividades de enfermagem. A Figura 3 demonstra a tela de cadastro das intervenções de enfermagem. 
Figura 3 - Tela de cadastro das prescrições de Enfermagem.

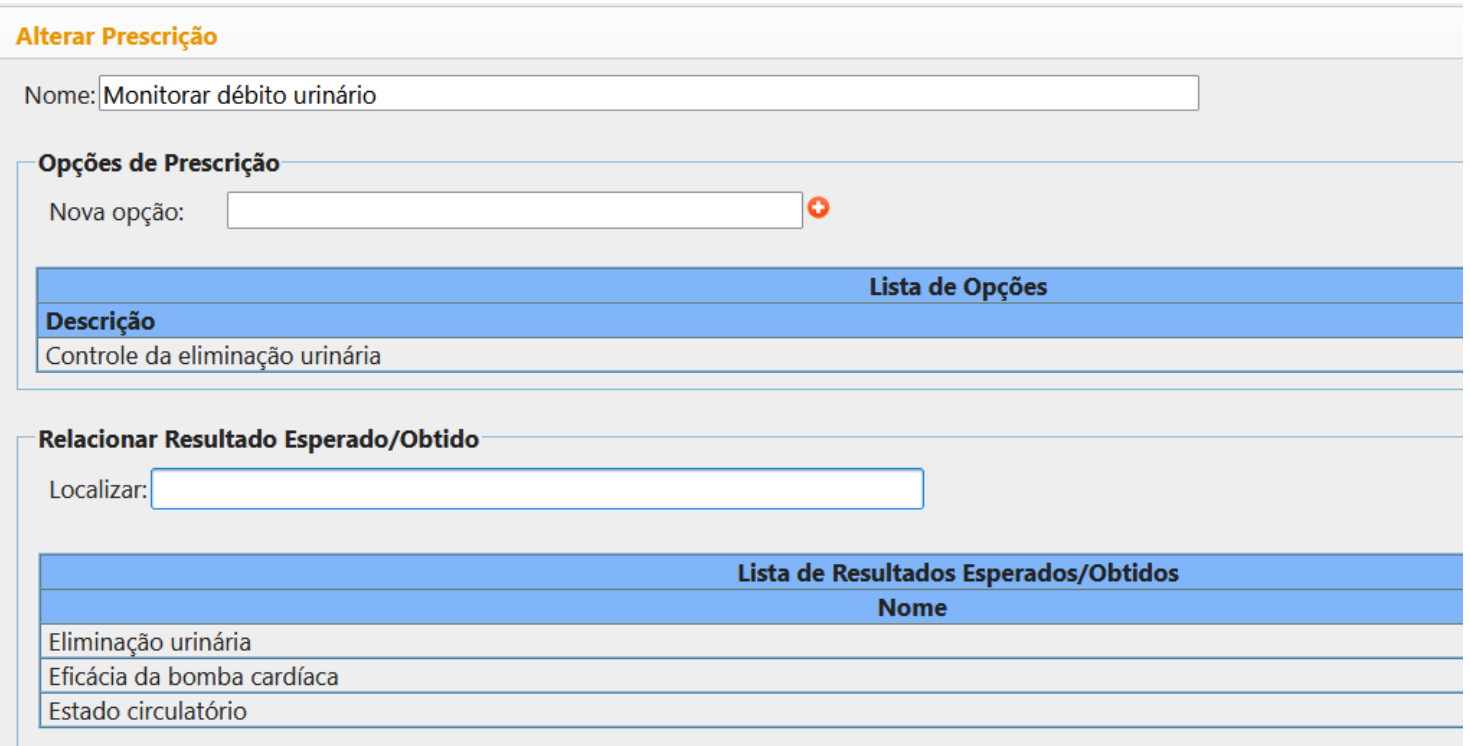

Fonte: SISPE (2021).

$\mathrm{Na}$ etapa seguinte foram cadastrados vinte e nove diagnósticos de enfermagem com seus títulos e definições. Os diagnósticos foram mapeados de acordo com os dados que compõem a coleta de dados. Para cada grupo de NHB foram previstas possibilidades de diagnósticos relacionados às evidências ou aos eventuais fatores de risco presentes.

Inseriu-se, para cada diagnóstico, as intervenções/atividade de enfermagem relacionadas, instituindo assim a ligação entre diagnósticos, intervenções e prescrições de enfermagem, conforme demonstrado pela Figura 4.

Figura 4 - Tela de cadastro dos diagnósticos de enfermagem.

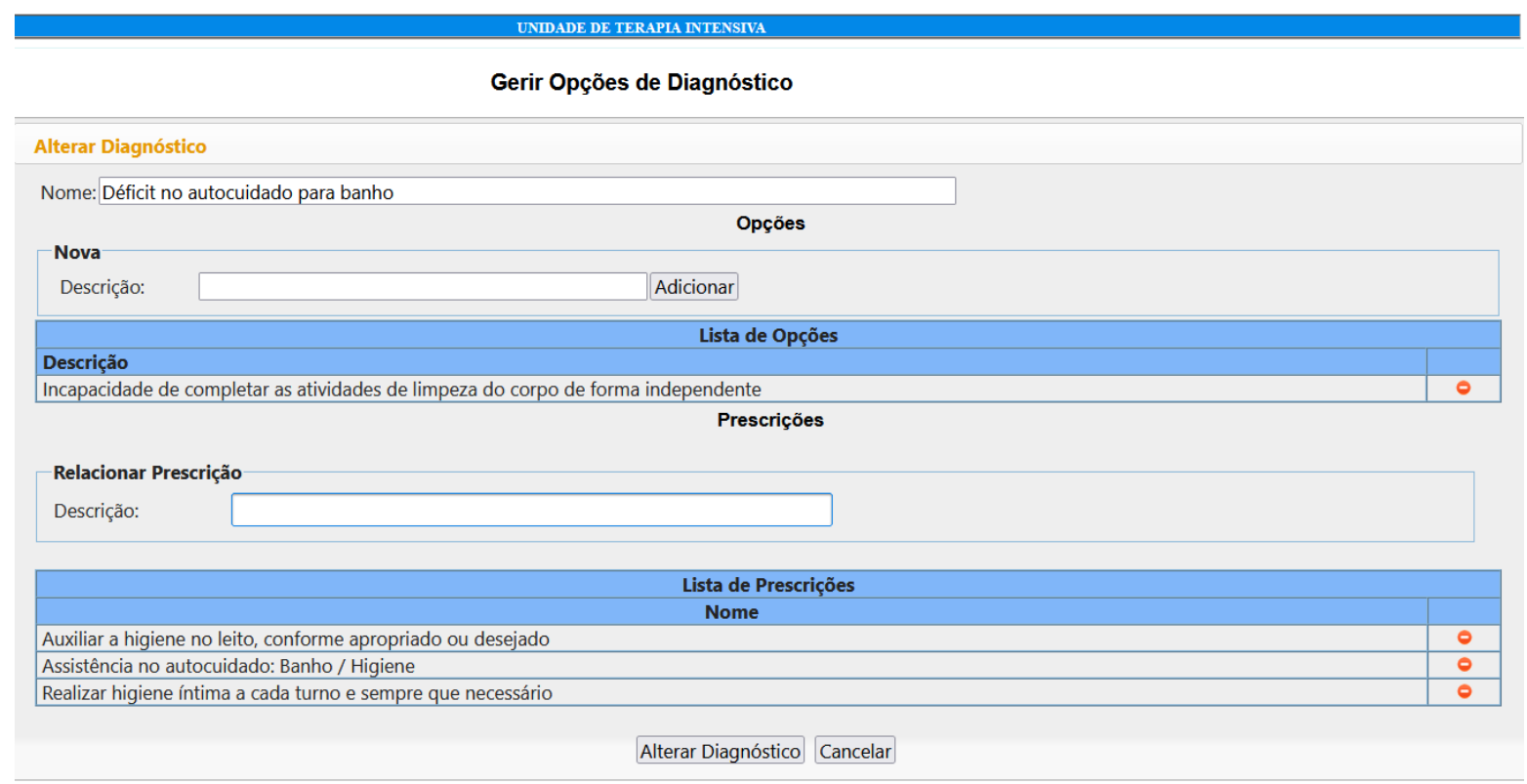

Fonte: SISPE (2021).

Na última fase da parametrização foram criados campos para registro da anamnese do paciente, agrupados de acordo com as necessidades humanas básicas: circulação, eliminação, higiene, integridade tecidual, nutrição/hidratação, percepção sensorial, termorregulação e oxigenação. 
Finalizada a parametrização, o sistema foi testado e verificou-se que se o SISPE foi capaz de estabelecer o relacionamento entre as fases do PE por meio das linguagens padronizadas, o que confirmou sua habilidade em permitir o registro do PE segundo suas etapas interdependentes e inter-relacionadas.

Uma revisão integrativa sobre o uso do processo de enfermagem aplicado a softwares incluiu 26 (vinte e seis) artigos e dentre esses apenas dois citam a utilização de um referencial teórico como suporte para o sistema (Domingos et al., 2017).

De acordo com resolução 358 de 2009 do Conselho Federal de Enfermagem, o PE deve estar fundamentado em um suporte teórico que oriente todas as etapas. As teorias de enfermagem são visões acerca de determinados fenômenos que se formam a partir das experiências do cotidiano. Contêm conceitos que representam os fenômenos pertinentes à ciência da enfermagem e auxiliam o enfermeiro na obtenção de dados relacionados às necessidades do paciente (Barros \& Lemos, 2017).

Em um sistema de informação elaborado para registro do PE em terapia intensiva utilizou-se em sua parametrização a Teoria das Necessidades Humanas Básicas, os diagnósticos de enfermagem da Nanda-I e as intervenções de enfermagem da NIC, contemplando três fases do PE (Martins \& Chianca, 2016).

Um website educacional elaborado para acadêmicos e enfermeiros, contém informações sobre o processo de enfermagem em cardiologia, e inseriu em seu conteúdo as cinco etapas do PE. Porém os autores descrevem apenas a utilização da linguagem padronizada da Nanda-I (Barros, Amâncio \& Ferreira, 2017).

Ainda, um Sistema eletrônico de Apoio à Decisão (SAD) elaborado para documentação em enfermagem nas unidades de clínica médica e cirúrgica de um hospital universitário contemplou as cinco fases do PE e utilizou as taxonomias da NandaI, NIC e NOC (Peres, Jensen \& Martins, 2016).

Os sistemas de classificação são instrumentos que aumentam a confiabilidade, validade e usabilidade da documentação em enfermagem. Quando usados em sistemas informatizados de documentação permitem o uso dos dados relativos aos cuidados de enfermagem para avaliar a assistência prestada e apoiar decisões de natureza clínica e gerencial (Peres, Lima \& Oliveira, 2013).

Barros e Lemos (2017) identificaram em alguns estudos o uso do PE de modo fragmentado, sendo que a etapa mais ausente foi a da avaliação de enfermagem. Os autores reforçam que o PE está dividido em fases que são inter-relacionadas, interdependentes e recorrentes, de modo que um software que não contemple todas as fases poderá comprometer a realização plena e efetiva do PE.

\subsection{Usabilidade do Sistema de Processo de Enfermagem}

A usabilidade do SISPE foi medida pela aplicação da System Usability Scale (SUS), que se utiliza de uma escala tipo likert com dez afirmações: cinco positivas e cinco negativas intercaladas. A soma das contribuições de cada item produz um único índice que irá representar a medida composta da usabilidade do sistema. Sua pontuação geral pode variar de 0 a 100.

A usabilidade é a característica que garante ao sistema de informação facilidade, adequação e aprendizagem rápida no modo de manejar este sistema, de forma que minimiza esquecimentos ou erros operacionais. Dessa forma, contribui para o alcance de maior eficiência e segurança na implementação das atividades as quais se destina, tornando essas atividades mais agradáveis para o usuário, garantindo a inteligibilidade, apreensibilidade e operabilidade (Rosa, 2019).

Neste processo são avaliados cinco componentes de qualidade, definidos por Tenório et al. (2011): capacidade de aprendizagem, eficiência, memorização, existência de erros, satisfação do usuário. Esses pontos são essenciais para a indicação da qualidade de um software, visto que a satisfação do usuário é imprescindível para o bom resultado do produto final.

A escala SUS classifica o sistema avaliado em seis categorias, de acordo com a pontuação obtida: pior impossível (025), ruim (26-39), aceitável (40-52), bom (53-74), excelente (75-85) e melhor impossível (86-100) (Bangor, Kortum \& Miller, 2009). 
Pesquisadores equipararam o SUS Score a valores relativos, sendo a mediana determinada em 68 . Valores acima de 68 estão superiores à média e valores inferiores estão abaixo da média (Bangor, Kortum \& Miller, 2008). Diante da pontuação encontrada foi possível fazer a classificação do SISPE quanto à usabilidade.

Neste estudo, a pontuação da escala SUS para o SISPE variou entre 62,5 e 100, de acordo com a avaliação dos enfermeiros e acadêmicos, conforme descrito na Figura 5.

Figura 5 - Pontuação da System Usability Scale por enfermeiros e acadêmicos.

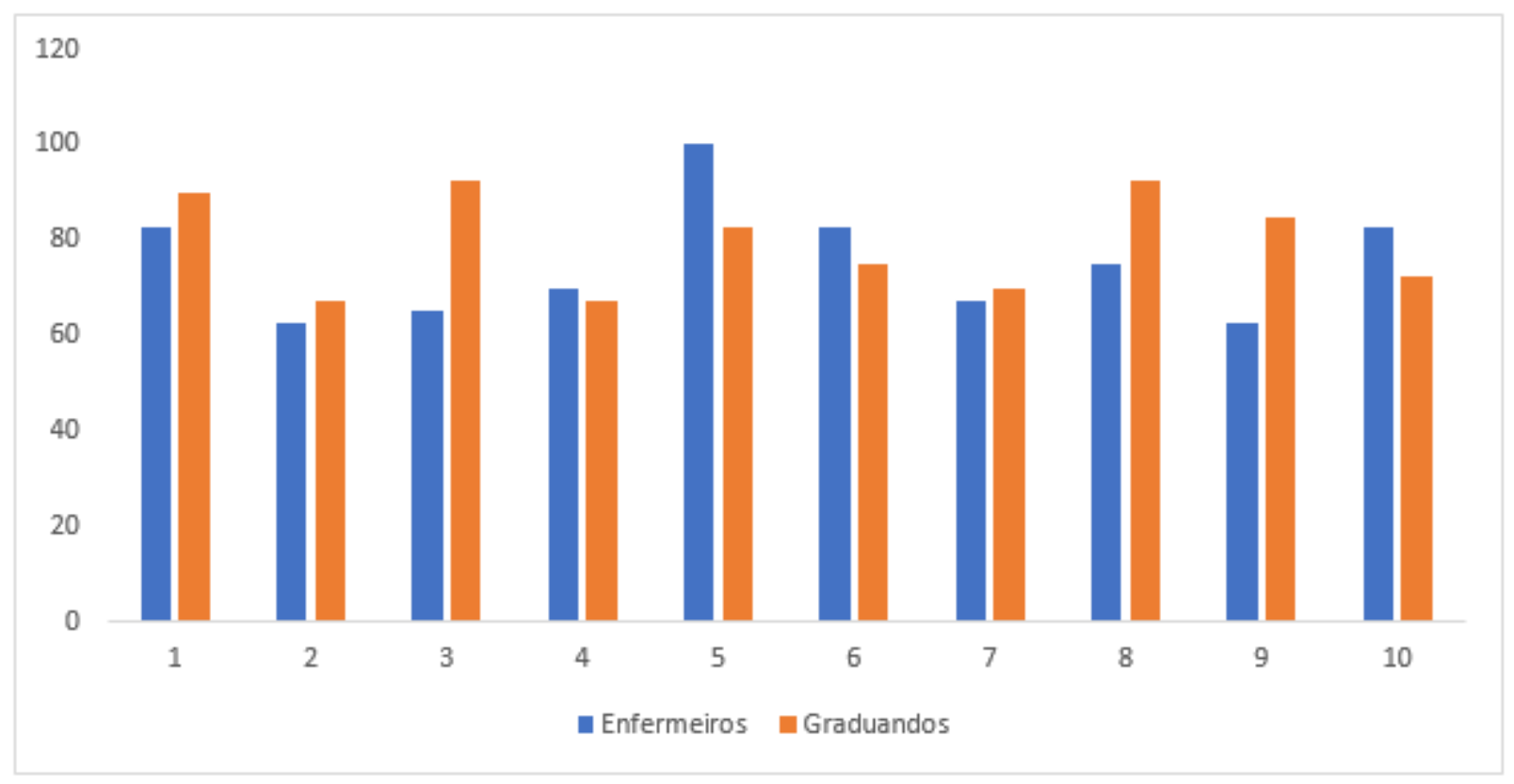

Fonte: Autores (2021).

A pontuação média para todos os participantes foi de 77,2 pontos, o que equivale à categoria de usabilidade excelente. A média da avaliação do grupo dos enfermeiros foi de 75, sendo a menor pontuação 62,5 e a maior 100; no grupo dos acadêmicos a menor pontuação foi 67,2 e a maior 92,5 com média equivalente a 79,5 pontos. Individualmente, nove participantes avaliaram o sistema como bom (62,5-72,5), sete como excelente (75-85) e três como melhor impossível (92,5-100).

Em pesquisa desenvolvida por Costa et. al (2021) foi realizada a análise da usabilidade do sistema WEB para gestão acadêmica Q-acadêmico, por meio da System Usability Scale, com o intuito de compreender as percepções dos usuários diante da utilização do sistema. Cento e um usuários responderam ao questionário atingindo a pontuação de 67,2, demonstrando que o escore de usabilidade do SISPE encontra-se acima desta média.

Augusto et al. (2021) desenvolveram e validaram um aplicativo para registro eletrônico do atendimento pré-hospitalar, sendo este avaliado por 25 participantes. A usabilidade do sistema foi verificada pela SUS e obteve como escore 66,7 pontos e o método SUS foi eficiente para avaliar a usabilidade pela percepção do usuário, o que corrobora cos os achados deste estudo.

Ao avaliar a usabilidade do SISPE os participantes deste estudo demonstraram satisfação com o seu desempenho tendo em vista a facilidade com que puderam realizar o registro do PE e a eficiência do sistema em cumprir as etapas necessárias para tal objetivo. A usabilidade do SISPE foi avaliada pelos participantes como excelente, o que demonstra ser viável a sua utilização por enfermeiros e estudantes, para o registro do processo de enfermagem. 


\section{Conclusão}

De acordo com os objetivos propostos e os resultados obtidos na presente pesquisa, concluiu-se que o SISPE vinculou as etapas do PE pela inserção das linguagens padronizadas da enfermagem, estabelecendo relacionamentos entre essas, e o resultado da parametrização do SISPE possibilitou o registro do PE. O escore de usabilidade apontado por enfermeiros e acadêmicos de enfermagem foi de 77,2 pontos, o que corresponde à categoria de usabilidade excelente.

Recomenda-se o desenvolvimento de instrumentos tecnológicos que alinhem o conhecimento teórico-prático da enfermagem. Novas pesquisas sobre o PE informatizado poderão confirmar os achados deste estudo em ambiente clínico e de ensino e assim contribuir para a efetivação do registro do processo de enfermagem.

\section{Referências}

Augusto, E. A. F., Werneck, A. L.., Rodrigues, L. C.., Ribeiro, R. de C. H. M.., Paschoal, V. D. A., \& Pompeo, D. A. (2021). Desenvolvimento e avaliação da usabilidade de um aplicativo para registro eletrônico de atendimento pré-hospitalar. Research, Society and Development, 10 (13), e463101321546. https://doi.org/10.33448/rsd-v10i13.21546.

Bangor, A., Kortum, P. T., \& Miller, J. T. (2008). An empirical evaluation of the System Usability Scale. International Journal of Human-Computer Interaction, 24(6), 574-594. https://psycnet.apa.org/record/2008-17901-003.

Bangor, A., Kortum, P. T., \& Miller, J. T. (2009). Determining What Individual SUS Scores Mean: Adding an Adjective Rating Scale. Jornal of Usability Studies, 4(3), 114-123. https://uxpajournal.org/wp-content/uploads/sites/7/pdf/JUS_Bangor_May2009.pdf.

Barros, A. L. B. L., Silva, V.M., Santana, R. F., Cavalcante, A. M. R. Z., Vitor, A. F., Lucena, A. F., et al. (2020). Brazilian Nursing Process Research Network contributions for assistance in the COVID-19 pandemic. Revista Brasileira de Enfermagem, 73(2), 1-12. https://doi.org/10.1590/0034-7167-2020-0798.

Barros, K. \& Lemos, I. (2017). Processo de Enfermagem: Fundamentos e Discussão de Casos Clínicos. Atheneu. http://revista.cofen.gov.br/index.php/enfermagem/article/view/1033/383

Barros, F. R. B., Amâncio, C. V., \& Ferreira, M. D. S. (2017). Desenvolvimento de um website educacional para o ensino do processo de enfermagem em cardiologia. Enfermagem em Foco, 8(2), 67-71. http://revista.cofen.gov.br/index.php/enfermagem/article/view/1033/383.

Brooke, J. (1996). SUS - A quick and dirty usability scale. Usability Evaluation in Industry, 189, 4-7. https://hell.meiert.org/core/pdf/sus.pdf.

Bulecheck, G. M., Butcher, H. W., Dochteman, J. M. \& Wagner, C. M. (2016). Classificação das Intervenções de Enfermagem (NIC). (6a. Ed). Rio de Janeiro: Elsevier.

Conselho Federal de Enfermagem (2009). Resolução nº 358/2009, de 15 de outubro de 2009. Dispõe sobre a Sistematização da Assistência de Enfermagem e a implementação do Processo de enfermagem em ambientes, públicos ou privados, em que ocorre o cuidado profissional de Enfermagem, e dá outras providências. Diário Oficial da União. Brasília, DF: Conselho Federal de Enfermagem. http://www.cofen.gov.br/resoluo-cofen-3582009_4384.html

Costa, R., Santos, A., \& Santiago, C. (2021). Análise de Usabilidade do Sistema Q-Acadêmico Utilizando o Método System Usability Scale (SUS): Um Estudo de Caso. In Anais do XIV Encontro Unificado de Computação do Piauí e XI Simpósio de Sistemas de Informação, (pp. 231-238). SBC. doi:10.5753/enucompi.2021.17776.

Domingos, C.S., Boscarol, G. T., Brinati, L M., Dias, A. C., Souza, C. C. \& Salgado, P. O. (2017). A aplicação do processo de enfermagem informatizado: revisão integrativa. Enfermería Global, 48, 620-36. https://scielo.isciii.es/pdf/eg/v16n48/pt_1695-6141-eg-16-48-00603.pdf.

Guimarães, J. O., Santos, L. N., Barreto, P. L., Melo, L. S., Andrade, J. S., Silva, F. J. C. P. (2020). Panorama do processo de enfermagem no brasil: uma revisão integrativa. Revista Enfermagem Atual In Derme, 94(32), e-020080. https://doi.org/10.31011/reaid-2020-v.94-n.32-art.926.

Herdman, T. H.\& Kamitsuru, S. (2018). Diagnósticos de Enfermagem da NANDA-I: Definições e classificações 2018-2020. (11a ed). Artmed.

Moorhead, S., Johnson, M., Maas, M. \& Swanson, E. (2016). Classificação dos Resultados de Enfermagem (NOC). (5a ed). Elsevier.

Oliveira, M. C. d. P., Moura, A. K. d., Lima, K. M. O., Medeiros, M. C. W. C. d., Lira, M. N., \& Lima, J. R. d. (2021). Construção de um protótipo de aplicativo móvel para processo de enfermagem do paciente renal. Research, Society and Development, 10(3). https://redib.org/Record/oai_articulo3116245constru\%C3\%A7\%C3\%A3o-de-um-prot\%C3\%B3tipo-de-aplicativo-m\%C3\%B3vel-para-processo-de-enfermagem-do-paciente-renal.

Peres, H. H. C., Jensen, R. \& Martins, T. Y. C. (2016). Avaliação da acurácia diagnóstica em enfermagem: papel versus sistema de apoio à decisão. Acta Paul. Enfermagem, 29(2), 218-224. https://www.scielo.br/j/ape/a/QxbMrpzFJ9ySL9ZxsyDLrRH/abstract/?lang=pt\#.

Peres, H. H. C., Lima, A. F. C. L. \& Oliveira, N. B. O. (2013). Implementação do diagnóstico de enfermagem no registro eletrônico de saúde. In: Nanda International Inc: Herdman, T. H. (Org.). PRONANDA - Programa de Atualização em Diagnósticos de Enfermagem - Conceitos Básicos (pp.65-85). Porto Alegre: Artmed Panamericana.

Polit, D.F \& Beck, C.T. (2019). Fundamentos de Pesquisa em Enfermagem: avaliação de evidências para a prática da enfermagem. (9a. ed). Artmed. 
Research, Society and Development, v. 11, n. 3, e1811326088, 2022

(CC BY 4.0) | ISSN 2525-3409 | DOI: http://dx.doi.org/10.33448/rsd-v11i3.26088

Rosa, S. C. S. (2019). Avaliação da usabilidade do módulo de sistematização da Assistência de Enfermagem do GSUS. (Dissertação de Mestrado). Universidade Federal do Paraná - UFPR, Curitiba, PR, Brasil. https://acervodigital.ufpr.br/bitstream/handle/1884/66632/R\%20-\%20D\%20\%20STELLAMARIS\%20CORDEIRO\%20SILVESTRE\%20ROSA.pdf?sequence=1\&amp;isAllowed=y

Souza, D. J. R. C. (2016). SISPE: Sistema de processo de Enfermagem voltado à evolução de paciente de acordo com os protocolos NANDA, NIC e NOC. (Monografia de Graduação em Ciências da Computação). Universidade Federal de Sergipe - UFS, São Cristóvão, SE, Brasil.

Tenório, J. M., Cohrs, F. M., Sdepanian, V. L., Pisa, I, T. \& Marin, H. F. (2011). Desenvolvimento e Avaliação de um Protocolo Eletrônico para Atendimento e Monitoramento do Paciente com Doença Celíaca. Revista de Informática Teórica e Aplicada,17(2), 210-220. https://seer.ufrgs.br/rita/article/view/rita_v17_n2_p210. 